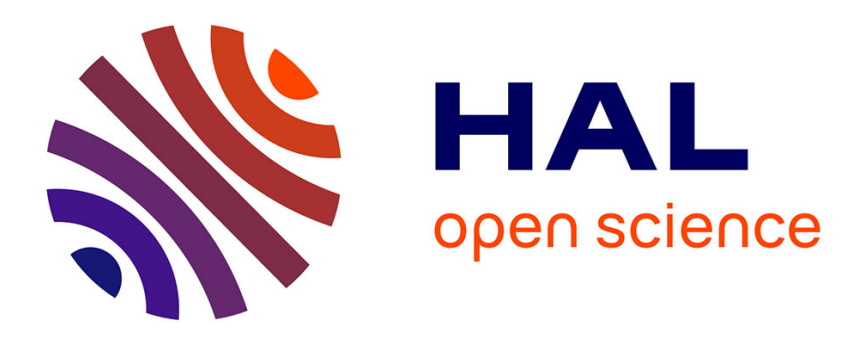

\title{
Panic in the movies ou deux ou trois formes de la panique dans le cinéma apocalyptique Hugo Clemot
}

\section{To cite this version:}

Hugo Clemot. Panic in the movies ou deux ou trois formes de la panique dans le cinéma apocalyptique. Sens-Dessous, 2015, 15, pp.83-90. 10.3917/sdes.015.0083 . hal-03510000

\author{
HAL Id: hal-03510000 \\ https://hal.science/hal-03510000
}

Submitted on 14 Jan 2022

HAL is a multi-disciplinary open access archive for the deposit and dissemination of scientific research documents, whether they are published or not. The documents may come from teaching and research institutions in France or abroad, or from public or private research centers.
L'archive ouverte pluridisciplinaire HAL, est destinée au dépôt et à la diffusion de documents scientifiques de niveau recherche, publiés ou non, émanant des établissements d'enseignement et de recherche français ou étrangers, des laboratoires publics ou privés. 
"Panic in the movies ou de deux ou trois formes de la panique dans le cinéma apocalyptique »

\section{Hugo Clémot}

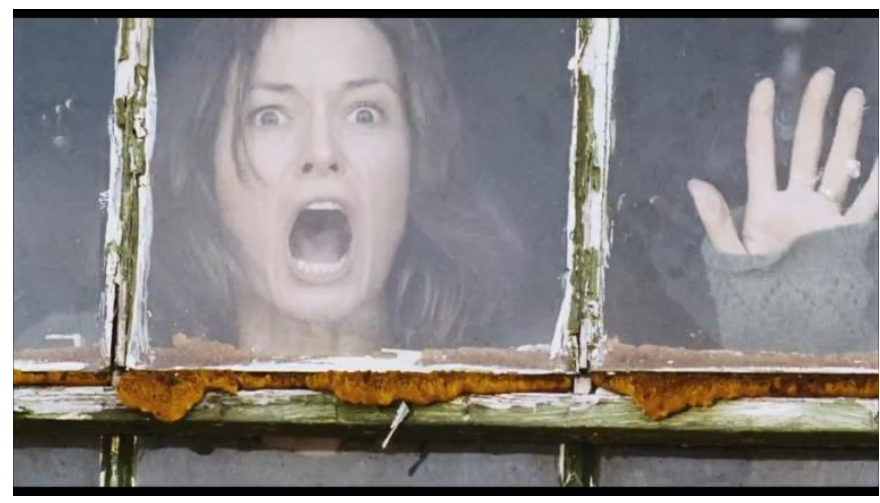

Juan Carlos Fresnadillo, 28 semaines plus tard, 2007

Plusieurs œuvres télévisuelles et cinématographiques à gros budget récentes, qui relèvent du genre de l'horreur épidémique1, comme The Walking Dead (série TV, Darabont et Kirkman, 2010auj.), Contagion (Soderbergh, 2011) ou World War Z (Forster, 2013) flattent le goût contemporain pour la mise en scène de la panique. Cela soulève une double question : quel rapport existe-t-il entre ce phénomène et ce genre cinématographique et pourquoi goûtons-nous ces histoires de panique?

\section{La panique comme affolement}

Le film 28 days later (28 jours plus tard, Boyle, 2002) et sa suite, 28 weeks later (28 semaines plus tard, Fresnadillo, 2007), ont eu un impact décisif sur cette mode qui consiste à filmer la panique collective, provoquée par l'arrivée de zombies plus ou moins rapides ${ }^{2}$. La première séquence de 28 weeks later mérite ainsi d'être évoquée pour relever certaines caractéristiques récurrentes des représentations cinématographiques de la panique ${ }^{3}$.

En nous montrant la paix d'un repas subitement rompue par l'arrivée de zombies qui entrent par les fenêtres de la maison, le film 28 weeks later nous offre une scène de panique exemplaire :

\footnotetext{
${ }^{1}$ Pour une justification de cette appellation, voir Clémot, «Une lecture des films d'horreur épidémique », Tracés. Revue de Sciences bumaines, $n^{\circ}$ 21, «Contagions », 2011/2, p. 167-184.

${ }^{2}$ Même si les hommes enragés de ces films ne sont pas vraiment des zombies, on fait généralement remonter à ces œuvres la vogue actuelle qui consiste à montrer des zombies aussi, voire plus rapides que des hommes. Voir Julien Bétan et Raphaël Colson, Zombies !, Lyon, les Moutons électriques, 2013 (2009), p. 272-275.

${ }^{3}$ Le cinéma ne fait donc pas seulement de la panique une forme d'anxiété profonde, à l'instar de la nosographie officielle. Dans ce qui suit, on cherchera à comprendre ce que nous voulons dire d'ordinaire quand nous employons ce terme, considérant que les problèmes conceptuels que pose la notion ne sauraient être résolus seulement par une référence scientifique.
} 
on y voit en effet surgir une peur collective très vive sur les visages de personnes dont l'esprit et le comportement sont si troublés qu'elles adoptent, pour la plupart, des conduites contraires à ce qu'aurait exigé leur survie, tant individuelle que collective. Mieux, en nous faisant passer brutalement d'une scène où l'on observe scrupuleusement les manières de table à une séquence où celui que l'on identifie rapidement comme le héros abandonne sa femme et un enfant aux dents des enragés, le film met simplement en évidence le trait essentiel de la panique selon les spécialistes : «l'effondrement soudain de l'ordre social ${ }^{4} »$.

À suivre ce trop bref résumé narratif des premières minutes du film, il semble que la panique soit essentiellement un affolement : elle rend fou sous l'effet d'une force elle-même irrationnelle, à la façon dont le dieu Pan pouvait, selon la mythologie grecque, rendre folles d'épouvante les nymphes qu'il poursuivait. Affolement, effroi, épouvante, il n'est pas étonnant que les films d'horreur constituent un médium privilégié pour l'expression et l'étude de la panique. On y découvre en effet que la panique prend des formes comportementales constantes, connues au moins depuis l'Antiquité, comme en témoigne les représentations sculpturales de l'épouvante des nymphes poursuivies par le Dieu Pan. Les expressions du visage qu'on peut y observer rappellent aussi, de façon frappante, un dessin de Le Brun pour illustrer l'effroi.

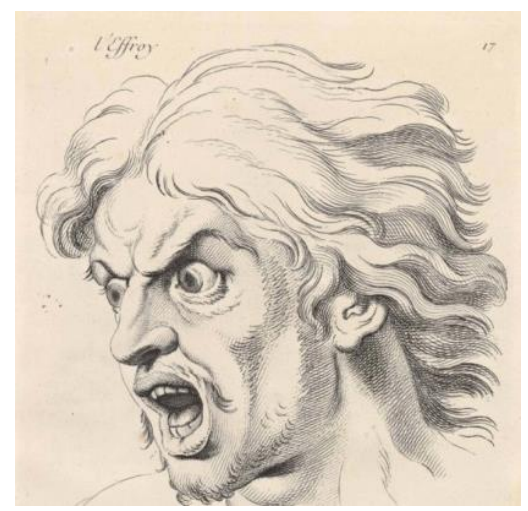

Le Brun, « L'effroi ${ }^{5} »$

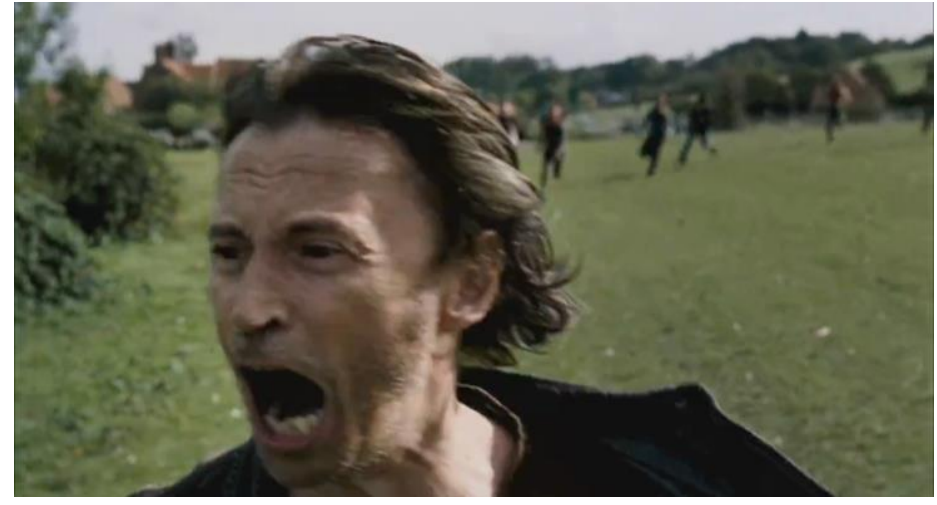

Juan Carlos Fresnadillo, 28 semaines plus tard, 2007

Cependant, outre les expressions comportementales et neurovégétatives (pâleur, sueurs, tachycardie, palpitations, tremblements, spasmes, dyspnée, bouche sèche), le cinéma d'horreur donne également accès aux manifestations subjectives de la panique. En effet, l'usage de la caméra portée, du faux raccord, de plans très brefs, le jeu sur les angles, les points de vue, la profondeur de champ, sans oublier la musique «post-rock» qui va crescendo, etc., font que le spectateur comprend assez mal ce qui se passe et se trouve au final presque aussi désorienté que

\footnotetext{
${ }^{4}$ Jean-Pierre Dupuy, La panique, Paris, Le Seuil, «Les empêcheurs de penser en rond », 2003, p. 16.

${ }^{5}$ Charles Le Brun, Les expressions des passions de l'âme, représentées en plusieurs testes gravées d'après les dessins de feu M. Le Brun, Paris, J. Audran, 1727.
} 
les personnages pour lesquels on l'incite à s’inquiéter. En paraphrasant Gombrich à propos du Cri (1895) de Munch, on pourrait dire que ce film nous fait vivre « la transformation de toutes nos sensations sous l'influence d'une émotion soudaine ${ }^{6} »$, la panique.

Mais si la panique peut faire qu'un homme abandonne femme et enfant, cela semble vouloir dire que l'irrationalité qu'elle révèle est intérieure à l'être humain qui, comme $\operatorname{Pan}^{7}$, contient son zombie potentiel, cette animalité, «cette sauvagerie, cette obscurité des profondeurs, qui ne demanderaient qu'à refaire surface en cas de crise grave ${ }^{8} »$ et à contaminer l'ensemble de la population : on pourrait définir la panique comme une

«peur collective intense, ressentie simultanément par tous les individus d'une population, caractérisée par la régression des consciences à un niveau archaïque, impulsif et grégaire, et se traduisant par des réactions primitives de fuite éperdue, d'agitation désordonnée, de violence ou de suicide collectif ${ }^{9}$ ».

À suivre cette définition, fidèle au mythe de Pan, de ce que l'on appelle «l'école française ${ }^{10}$ », on comprend donc en quoi l'irrationalité et le caractère contagieux de la panique en font l'émotion que cherche spécifiquement à susciter le genre cinématographique de l'horreur épidémique.

Cependant, si certains genres cinématographiques existent en relation avec une émotion que les films qui les composent sont censés susciter chez les spectateurs de façon privilégiée, alors il faut reconnaitre qu'il est un film qui est une sorte de matrice des films d'horreur épidémique, bien que ne s'y produise aucune épidémie et qui tendrait plutôt à vérifier la thèse de l'école américaine selon laquelle « [1]a panique est beaucoup moins irrationnelle et sauvage que le mythe la décrit ${ }^{11} »$ : Panic In The Year Zero (Panique année zéro, Ray Milland, 1962).

\footnotetext{
${ }^{6}$ Ernst Gombrich, Histoire de l'art, Paris, Phaidon, 2001, p. 564-566. Il est d'ailleurs remarquable que la séquence se termine sur un ponton, qui n'est pas sans rappeler la jetée du fameux tableau de Munch.

7 « Héritier direct de la nuit originelle, l'Arcadien a le privilège de pouvoir, à tout instant, revivre sa naissance à l'humanité. Il est, culturellement, sur le seuil. Un pas en avant, le voici tout à fait grec et même, ce qui est important aux yeux de l'histoire, démocrate ; un pas en arrière, le voilà redevenu sauvage. Cette position liminale lui vaut un certain prestige. » Philippe Borgeaud, Recherches sur le dieu Pan, Paris, Droz, 1982 ; cité par Dupuy, La panique, op. cit., p. 17.

${ }^{8}$ Dupuy, La panique, op. cit., p. 39.

${ }^{9}$ L. Crocq, C. Doutheau et M. Sailhan, «Les réactions émotionnelles dans les catastrophes », Encycl. méd. chir., Psychiatrie, 37113 D, 2, 1987.

${ }^{10}$ Voir Dupuy, La panique, op. cit., p. 30-31, qui rappelle l'influence déterminante pour l'école française des textes de Freud et Le Bon, respectivement: Freud, "Psychologie collective et analyse du moi », in S. Freud, Essais de psychanalyse, trad. fr. S. Jankélévitch, Paris, Payot, 1925 (1921) ; Gustave Le Bon, La Psychologie des foules, Paris, Alcan, 1895.

${ }^{11}$ Dupuy, La panique, op. cit., p. 39.
} 


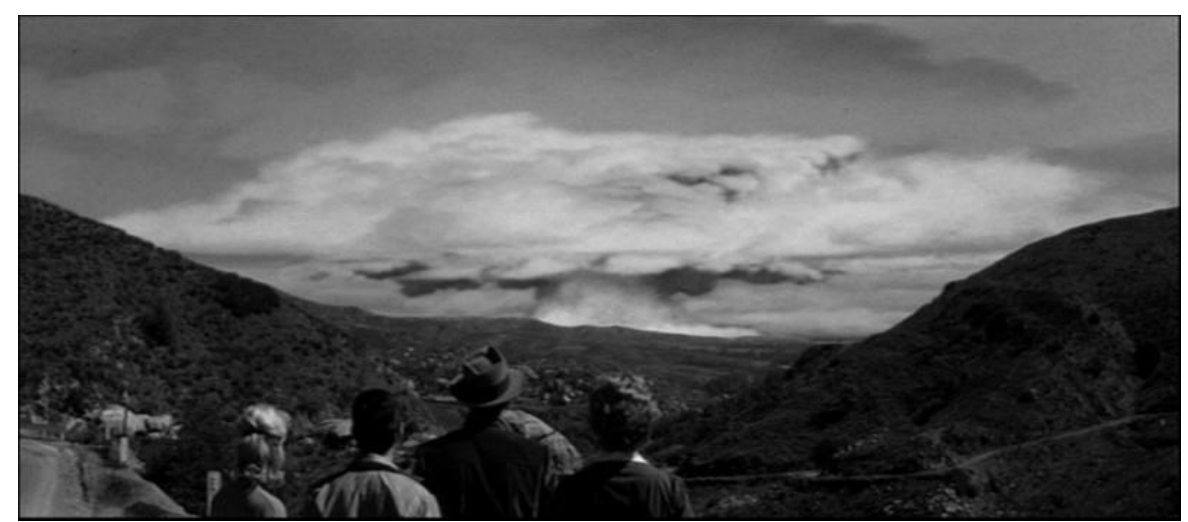

Panic In The Year Zero (Ray Milland, 1962)

\section{La rationalité de la panique}

Le film Panic In The Year Zero (PITYZ ensuite) nous permet de penser la notion de panique selon deux ou trois formes de première importance pour la subjectivité moderne. En effet, si le terme «panique» est d'abord utilisé par l'un des personnages pour expliquer la conduite automobile dangereuse des habitants de Los Angeles qui fuient la ville détruite par une bombe atomique, et donc employé en un sens qui renvoie à l'irrationalité d'une telle prise de risque, le film se concentre essentiellement sur le processus de désocialisation avec « désintégration des normes sociales ${ }^{12}$ » et «destruction des liens primaires les plus forts ${ }^{13}$ » que la destruction des institutions sociales et politiques encourage : l'absence de police, d'armée et même d'État, depuis que les moyens de communication ont été détruits, fait qu'il devient plus rationnel d'adopter la défiance, plutôt que la confiance envers les autres ${ }^{14}$. En effet, le choix de la sécurité individuelle conduit à faire défection, plutôt qu'à coopérer, un choix qui a un aspect contagieux dans la mesure où il peut suffire d'avoir été trahi une fois, ou même d'avoir observé une trahison, pour renoncer à la confiance et à la coopération ${ }^{15}$, ce qui produit une situation d'« absence totale de coordination, de coopération et de concertation entre les actions des uns et des autres, qui deviennent soumises à une logique purement individualiste ${ }^{16} »$, bien connue des philosophes depuis au moins Thomas Hobbes. La disparition des institutions politiques traditionnelles crée en effet un retour à l'état de nature au sens où, en l'absence de lois ${ }^{17}$, chacun a un droit illimité sur

\footnotetext{
${ }^{12}$ E.L Quarantelli, «The Nature and Condition of Panic », The American Journal of Sociology, vol. 9, n 3, 1954, p. 270. 13 Idem.

${ }^{14}$ Qu'on songe au dialogue suivant entre le père et la mère : « Mère : - Isn't there anybody we can trust? Père : - No, not yet.»

15 On pense à la scène de la station-service, au début du film.

${ }^{16}$ Dupuy, La panique, op. cit., p. 40

17 « No rules, no regulations, and no laws. », dit le fils à sa mère pour justifier le comportement de son père.
} 
toutes choses, ce qui ne peut conduire, par le jeu conjugué de la compétition, de la défiance et de la « gloire'18», qu'à «la guerre de chacun contre chacun ${ }^{19} »$. En suivant les efforts d'un père de famille pour protéger les siens par tous les moyens, le film montre bien comment la violence qu'il exerce n'est pas le résultat d'une régression irrationnelle au niveau de l'animal, mais celui d'un calcul rationnel de l'action la plus sûre ${ }^{20}$ dans un contexte de rareté des moyens de subsistance : «C'est l'accès limité aux ressources permettant d'échapper au désastre qui, faisant de chacun un obstacle ou un rival pour les autres, provoque la panique ${ }^{21}$.»

On objectera que le film fait tout pour montrer que la force du héros est qu'il ne cède justement pas à la panique, contrairement à tous ceux qui ne font que s'imiter sans raison en fuyant en automobile. Seulement, c'est oublier qu'il peut être rationnel d'imiter les autres dans une situation d'incertitude pratique où se posent des problèmes de coordination des actions individuelles. Par exemple, quand un mouvement de panique, où chacun chercherait à fuir d'un milieu clos et bondé, pourrait se produire, il est rationnel de chercher à anticiper cette fuite, ce qui peut avoir pour effet de provoquer l'événement auquel on cherchait à échapper : la panique est « une représentation sociale autoréalisatrice ${ }^{22}$ ». Il est remarquable que, si le héros de PITYZ ne fuit pas dans la même direction que la plupart des autres hommes, il n'en fuit pas moins lui aussi les radiations atomiques et surtout les comportements violents, rationnels et irrationnels, des autres, imitant en cela leur comportement général. En effet, «si je ne sais rien au sujet de la situation dans laquelle je me trouve (en situation de panique, par exemple), je peux me dire qu'il y a une chance que les autres sachent : à les imiter, je tirerai parti de leur savoir ${ }^{23}$ », ce qui fait dire à Keynes que la solution la plus rationnelle est de tenter « de nous conformer au comportement majoritaire ou moyen ${ }^{24} »$, et a conduit Descartes à adopter, comme première maxime de sa «morale par provision ${ }^{25}$ », de se conduire «suivant les opinions les plus modérées, et les plus éloignées de l'excès, qui fussent communément reçues en pratique par les mieux sensés de ceux avec lesquels j'aurais à vivre ${ }^{26}$. » Autrement dit, loin d'être seulement une forme de comportement irrationnelle, pathologique, l'imitation panique peut aussi être le résultat d'un calcul rationnel dont on peut trouver une description chez Hobbes :

\footnotetext{
${ }^{18}$ Hobbes, Léviathan, I, Chapitre XIII.

${ }^{19} \mathrm{Idem}$.

${ }^{20}$ On pense au braquage du marchand d'armes, à la destruction du barrage routier, etc. Jean-Pierre Dupuy parle d’une « rationalité limitée » ou «située » pour désigner cette situation où « [l]'individu ne régresse pas au niveau de l'animal, il n'est pas soumis à ses instincts les plus primitifs, il conserve une certaine lucidité, la capacité d'apprécier la situation et de prendre une décision. » Dupuy, La panique, op. cit., p. 41.

${ }^{21}$ Dupuy, La panique, op. cit., p. 42.

${ }^{22}$ Dupuy, La panique, op. cit., p. 43.

${ }^{23}$ Dupuy, La panique, op. cit., p. 114-115.

${ }^{24}$ J. M. Keynes, "The General Theory of Employment », The Quarterly Journal of Economics, vol. 51, 1937 ; cité par Dupuy, p. 115.

${ }^{25}$ Descartes, Discours de la Méthode, troisième partie, Paris, GF, 1966, p. 51.

${ }^{26}$ Idem.
} 
«La crainte dont on n'aperçoit pas le pourquoi ou l'objet est appelée terreur panique ; on la nomme ainsi d'après les fables qui font de Pan son auteur : en réalité, celui qui ressent le premier une telle frayeur en aperçoit toujours de quelque façon la cause; mais les autres s'enfuient à cause de l'exemple, chacun supposant que son voisin sait pourquoi. C'est pourquoi cette passion n'atteint personne si ce n'est dans une foule, une multitude ${ }^{27}$.»

Dans son livre de référence ${ }^{28}$, Jean-Pierre Dupuy consacre plusieurs chapitres à montrer en quoi cette rationalité est aussi celle de la panique financière et il n'est pas très étonnant de retrouver dans PITYZ plusieurs références à l'impact de la crise panique sur la valeur de l'argent. Cependant, une lecture attentive du film permet de saisir que l'enjeu d'une juste compréhension du phénomène de la panique n'est pas seulement économique, ni même social, mais surtout proprement philosophique en tant qu'il a à voir avec la subjectivité moderne.

\section{La panique ordinaire et ses remèdes}

Il y a une tension dans le texte de Hobbes quant à la définition donnée de la terreur panique : ce serait "une crainte dont on n'aperçoit pas le pourquoi ou l'objet», sauf quand on est le premier à la ressentir. Si l'on comprend bien qu'il s'agit pour Hobbes de donner une définition génétique qui rende compte du caractère contagieux de la panique, il faut néanmoins voir, dans sa concession initiale, la reconnaissance de ce que la panique peut aussi et, peut-être même, doit d'abord être individuelle ${ }^{29}$.

Quand on pense à PITYZ, on s'aperçoit que la panique qui nous est montrée est surtout celle du père de famille, dont on pourrait dire qu'il est dans une situation de scepticisme pratique radical, c'est-à-dire d'incertitude quant à ce qu'il doit faire pour protéger les siens, mais aussi de pleine conscience de la nécessité de sortir sans attendre de cette irrésolution. Dans ce film, la panique est donc l'émotion qui saisit celui qui croit qu'il ne peut avoir aucune certitude mais qui est certain qu'il doit agir vite, sans attendre, pour survivre. C'est donc l'émotion qui accompagne de façon privilégiée l'expérience du scepticisme pratique, qui semble propre au sujet moderne, au moins depuis Descartes.

Pour sortir de cette irrésolution, il est au moins deux ou trois options. On peut agir dans la précipitation et en s'en remettant entièrement, d'une façon d'autant moins critique que l'on

\footnotetext{
${ }^{27}$ Hobbes, Léviathan, première partie, chapitre vi (trad. F. Tricaud).

${ }^{28}$ Dupuy, La panique, op. cit.

${ }^{29}$ N'en déplaise à Jean-Pierre Dupuy, La panique, op. cit., note 1, p. 19.
} 
cherche à étouffer le scepticisme qui accompagne d'ordinaire la réflexion, aux préjugés majoritaires : si le point de départ d'un tel comportement mimétique peut être rationnel, il verse rapidement, on s'en doute, dans l'irrationalité et conduit assez directement à la catastrophe.

On peut aussi choisir, à la manière de Descartes, de demeurer sceptique, tout en adoptant une «morale par provision», c'est-à-dire en attendant d'avoir trouvé mieux, en l'occurrence des certitudes, une morale définitive, représentée dans le film par le retour espéré des autorités et, avec elles, de la «civilisation » tenue pour le seul fondement possible d'un retour à la coopération et à la confiance. Cependant, comme dans de nombreux films d'horreur épidémique ou apocalyptiques et malgré les principes qu'il cherche à justifier rationnellement ${ }^{30}$, le héros va faire l'expérience d'une extension et d'une intensification de son scepticisme en même temps que de la panique concomitante. En effet, d'abord relatif aux institutions politiques, économiques et sociales, le scepticisme du héros va porter sur la bonté de l'espèce humaine, avant que sa femme elle-même ne s'aperçoive qu'elle ne savait pas jusqu’ici qui était réellement son mari : «After all these years, I thought I knew you, but you turn out to be a stranger. Robbing and mauling people like some kind of a cheap hoodlum. » Cette expérience pratique, qui n'est pas si extraordinaire, du scepticisme à propos d'autrui va finalement conduire le mari, dans un moment d'abattement qui est, pour lui, une façon de paniquer, au scepticisme quant à sa propre identité, lorsqu'il va s'apercevoir qu'à force de chercher le pire chez les autres, il l'a trouvé en lui-même, en tuant deux hommes ${ }^{31}$. D'un point de vue philosophique, l'histoire du personnage peut s'interpréter ainsi: désespérant d'atteindre le fondement rationnel espéré, l'esprit désireux de certitudes peut finalement céder à une panique de second ordre quand il s'aperçoit qu'il a détruit par sa défiance tout ce qui avait de l'importance et que son enquête était censée permettre de retrouver. La leçon est la même que celle que Stanley Cavell a tiré de son étude d'Othello de Shakespeare ${ }^{32}$ : le désir de connaissance intime, exclusive du monde et de l'autre est mortel ; plus on cherche à s'assurer de la présence du monde et que l'on a raison d'avoir confiance en l'autre, plus l'objet désiré s'éloigne et plus le scepticisme grandit.

Pour sortir de l'irrésolution et ne pas céder à la panique, on peut enfin pratiquer des exercices spirituels du type de ceux que préconise Épicure, par exemple, dans sa fameuse Lettre à Ménécée pour atteindre le bonheur, c'est-à-dire l'absence de troubles. On pourrait en effet relire l'ensemble de la Lettre comme une liste de remèdes pour se soigner du trouble de l'âme en quoi consiste la

\footnotetext{
${ }^{30}$ L'échange suivant entre le père et la mère illustre bien l'idée la rationalité de la panique : « Mère : Intelligent people don't just turn their backs on the rest of the world. Père: Under these conditions, intelligent people would be the first to try. »

${ }^{31} \mathrm{Il}$ y aurait ici un parallèle à établir avec le film Carriers (Pastor, 2010), un film d'horreur épidémique sans monstre mais qui montre la fabrique du monstrueux à partir de l'humain. Voir Clémot, "Une lecture des films d'horreur épidémique », art. cit., p. 179.

${ }^{32}$ Cavell, Le déni de savoir dans six pièces de Shakespeare, Paris, Seuil, 1993.
} 
panique, à commencer par le premier, l'idée que les dieux, y compris le dieu Pan, ne sont pas à craindre. En effet, il sera souvent possible de se libérer de la panique produite par l'impression qu'il ne peut y avoir aucune certitude sinon celle qu'il faut agir sans attendre, en interrogeant les raisons qui poussent à adopter ce scepticisme et à se croire dans une situation d'urgence, plutôt que « tranquille, comme un ancien, face à l'avenir ${ }^{33} »$.

Cependant, au lieu de présupposer qu'il doit toujours être possible de cesser de paniquer en connaissant mieux le monde et les mauvaises raisons qui troublent notre âme, il faudra admettre cette vérité du scepticisme qui est que nous devons reconnaittre le caractère séparé du monde et des autres, c'est-à-dire l'idée que nous ne pourrons jamais être absolument sûrs de les connaittre. Mais si la panique est le sentiment qui peut accompagner l'expérience du scepticisme pratique dans une situation d'urgence, alors cela veut dire qu'il nous faut reconnaître que la panique menace nécessairement notre existence, que notre vie la contient à titre de composant émotionnel constitutif avec lequel il nous faut apprendre à vivre, comme l'ingrédient nécessaire d'une recette que nous devons savoir cuisiner. Vivre avec le scepticisme, contenir la panique au sens où l'on parvient à en limiter les conséquences désastreuses, supposera donc d'apprendre à se passer de connaissance, c'est-à-dire renoncer à savoir que le monde existe, et simplement $l^{\prime} \ll$ accepter $^{34} »$ comme étant séparé de nous, renoncer à savoir que les autres existent, pour mieux s'efforcer de « reconnaître ${ }^{35}$ » leur présence en se révélant à eux, convaincu que « [c]'est la chose que nous ne savons pas qui peut nous $\operatorname{sauver}^{36}{ }^{2}$, c'est-à-dire privilégier la confiance et la coopération sur la défiance et la défection systématiques sans être sûrs que les autres coopéreront à leur tour. Selon le conseil d'Épicure, on pourra mettre en pratique ces remèdes avec ses proches, de façon à retrouver, par la force de rituels quotidiens partagés en commun, la tranquillité d'esprit qu'un tel usage du corps est en mesure de contribuer à apporter. C'est l'un des sens que l'on peut donner à ce qu'on pourrait appeler la «leçon de la caverne » de PITYZ, dispensée, avant le repas, par le père de famille :

«We don't know what lies abead of us. The unknown has always been man's greatest demoralizer. Maybe we can cope with this by maintaining our sense of values. By carrying out our daily routine, the same as we always have. Rick, for instance, and myself, will shave every day. Although, in his case, maybe every other day. These concessions of civilization are important. They are our links with reality. And because of them, we might be less afraid.»

\footnotetext{
${ }_{33}$ Épicure, Lettre à Ménécée, trad. O. Hamelin et J. Salem, Paris, Librio, 2009, p. 11.

${ }^{34}$ Cavell, «L'évitement de l'amour », Dire et Vouloir Dire, Paris, Cerf, 2009, p. 483.

${ }^{35}$ Idem. Voir surtout Stanley Cavell, «Connaitre et reconnaitre », DVD, op. cit., p. 377-412.

${ }^{36}$ Cavell, «L'évitement de l'amour », DVD, op. cit., p. 485.
} 
On pourrait aussi finalement donner un sens stö̈cien à cette expérience de retrouver la présence du monde et la confiance dans les autres que permettent certains gestes ordinaires quand on s'est rendu compte que rien ne garantit jamais (c'est là la vérité du scepticisme) que nous atteignions le monde ou que nous comprenions les autres. En effet,

"[q]ue nous le fassions en fin de compte est affaire de ce que nous partageons certains itinéraires d'intérêt et de sentiment, certains modes de réaction, certains sens de l'humour, de l'importance et de l'accomplissement, de ce qui est insupportable, de ce qui est comparable à quoi d'autre, de ce qu'est un reproche, de ce qu'est le pardon, des cas où tel énoncé est une affirmation, où il est un appel, où il est une explication - tout ce tourbillon de l'organisme que Wittgenstein appelle des «formes de vie ». La parole et l'activité humaines, leur santé mentale et leur communauté ne reposent sur rien de plus que cela, mais sur rien de moins non plus ${ }^{37}$.»

Que cette « vision » soit propre à susciter la panique est ce qui vient vérifier l'idée que la panique est le sentiment qui accompagne une expérience de scepticisme pratique, lorsqu'on s'aperçoit, après une catastrophe notamment, que ce que nous tenons pour humain ne repose sur aucun fondement rationnel absolu, mais seulement sur nos façons de vivre ou nos « formes de vie » ou encore notre «nature », si par nature on n'entend pas seulement ce qui en l'homme n'a pas été créé par lui, mais aussi tout ce qu'il est du fait de son intelligence, féconde et créatrice. On cesse de paniquer quand on s'aperçoit que ces «formes de vie» ne sont pas «rien non plus », que l'ordinaire, le quotidien, malgré la panique qu'il contient, contient aussi les moyens de sortir de l'irrésolution pratique en vivant une expérience de présence au monde et de confiance dans les autres que l'on peut bien qualifier de "panique », au sens où Pan était identifié par les stoïciens avec l'univers, à la nature intelligente, féconde et créatrice, au sens où Julien Gracq l'emploie quand il parle des « humbles besognes ${ }^{38} »$ d'antan « autour du puits et du lavoir » comme relevant de « ces gestes profonds qui font bondir au cœur comme un sentiment panique de la permanence de la vie » et qui renvoie, semble-t-il, à ce qu'il a appelé « le sentiment du oui ${ }^{39}$ ».

\footnotetext{
${ }^{37}$ Cavell, «La seconde philosophie de Wittgenstein est-elle à notre portée ? », DVD, op. cit., p. 138-139.

38 Julien Gracq, Le Rivage des Syrtes, Paris, Corti, 1951, p. 71.

39 «Oui, le monde extérieur existe pour moi. Et fortement. Et ce sentiment de très vigoureuse existence s'accompagne du sentiment du oui plutôt que du non. » Interview au Magazine littéraire, $\mathrm{n}^{\circ}$ 179, décembre 1981 ; cité par A. Chareyre-Méjan in Essai sur la simplicité d'être, Toulouse, éditions érès, coll. « 69 », 2009, p. 171.
} 